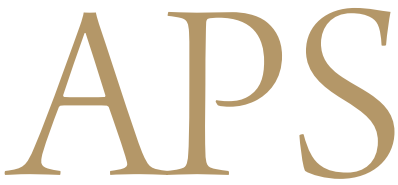

Archives of Plastic Surgery

\title{
Ultrasonic dissection versus electrocautery for immediate prosthetic breast reconstruction
}

\author{
Dongeun Lee, Bok Ki Jung, Tai Suk Roh, Young Seok Kim \\ Department of Plastic and Reconstructive Surgery, Gangnam Severance Hospital, Yonsei University College of Medicine, Seoul, Korea
}

Background Ultrasonic dissection devices cause less thermal damage to the surrounding tissue than monopolar electrosurgical devices. We compared the effects of using an ultrasonic dissection device or an electrocautery device during prosthetic breast reconstruction on seroma development and short-term postoperative complications.

Methods We retrospectively reviewed the medical records of patients who underwent implant-based reconstruction following mastectomy between March 2017 and September 2018. Mastectomy was performed by general surgeons and reconstruction by plastic surgeons. From March 2017 to January 2018, a monopolar electrosurgical device was used, and an ultrasonic dissection device was used thereafter. The other surgical methods were the same in both groups.

Results The incidence of seroma was lower in the ultrasonic dissection device group than in the electrocautery group (11 [17.2\%] vs. $18[31.0 \%] ; P=0.090)$. The duration of surgery, total drainage volume, duration of drainage, overall complication rate, surgical site infection rate, and flap necrosis rate were comparable between the groups. Multivariate analysis revealed that the risk of seroma development was significantly lower in the ultrasonic dissection device group than in the electrocautery group (odds ratio for electrocautery, 3.252; 95\% confidence interval, 1.242-8.516; $\mathrm{P}=0.016$ ).

Conclusions The findings of this study suggest that the incidence of seroma can be reduced slightly by using an ultrasonic dissection device for prosthesis-based breast reconstruction. However, further randomized controlled studies are required to verify our results and to assess the cost-effectiveness of this technique.

Keywords Breast / Mammaplasty / Breast implants / Electrocoagulation / High-intensity focused ultrasound ablation
Correspondence: Young-seok Kim Department of Plastic and Reconstructive Surgery, Gangnam Severance Hospital, Yonsei University College of Medicine, 211 Eonju-ro, Gangnam-gu, Seoul 06273, Korea Tel: +82-2-2019-1004 Fax: +82-2-2019-4833 E-mail: PSYSKIM@YUHS.AC

Received: June 13, $2019 \bullet$ Revised: September 2, $2019 \bullet$ Accepted: October 9, 2019

pISSN: 2234-6163 • elSSN: 2234-6171 • https://doi.org/10.5999/aps.2019.00759 • Arch Plast Surg 2020;47:20-25

\section{INTRODUCTION}

Seroma, which is one of the most common complications in prosthetic breast reconstruction, increases the incidence of other morbidities, such as surgical site infection, and the length of hospital stay. Of particular note, in patients with breast cancer, seroma development can lead to delays in the administration of adjuvant therapy. Several hypotheses have been proposed regarding seroma development. Seromas may be the consequence of surgical disruption of both the lymphatics and capillaries [1], injuries of the surrounding lymph vessels due to heat dispersion [2], and the consequence of inflammatory exudates [3]. In our 
opinion, all three of these hypotheses may be valid to some extent [4]. The causes of seroma development in breast reconstruction are thought to include the presence of a large irregular dead space, lymphatic disruption that occurs after total mastectomy, insertion of a foreign body, chest wall movement, and persistent inflammation in response to the tissue damage that occurs during the surgical process [1,4-6].

Monopolar electrosurgical devices have been frequently used for nearly 50 years. Fulguration, precise vaporization, and coaptation of large vessels are facilitated by using an electrosurgical device instead of a conventional scalpel; thus, bleeding is controlled relatively easily, which is advantageous for securing visibility and reducing operating time [7]. However, the use of a monopolar electrosurgical device is associated with the risk of seroma formation after surgery because it causes thermal damage to the surrounding tissue. Studies have reported that using a monopolar electrosurgical device resulted in an increase in the seroma formation rate and drainage volume compared to a conventional scalpel [8]. Therefore, various advantages and disadvantages are associated with the use of monopolar electrosurgical devices in breast surgery.

Recently, ultrasonic dissection devices have been introduced as an alternative. These devices use ultrasonic waves to dissect the tissue, making it possible to perform a more sophisticated dissection with a narrower circumference of collateral thermal damage to the surrounding tissue compared to the damage caused by monopolar electrosurgical devices [9]. Furthermore, bleeding control and dissection can be performed simultaneously, and there is a lack of surgical smoke and reduced charring; consequently, a clearer visual field can be secured. Studies have already reported that using an ultrasonic dissection device instead of an electrosurgical device for radical mastectomy was associated with a significant decrease in the incidence of seroma development [10-12].

To date, no study has investigated the surgical outcomes obtained by using an ultrasonic dissection device in comparison with those obtained by using a monopolar electrosurgical energy device during prosthetic breast reconstruction. Therefore, this study compared the effects of using an ultrasonic dissection device or a monopolar electrosurgical device during prosthesisbased breast reconstruction on seroma formation and shortterm postoperative complications.

\section{METHODS}

\section{Study design}

The study was conducted at the plastic surgery department of our hospital, and the study protocol was approved by the Insti- tutional Review Board of Gangnam Severance Hospital. Patients who underwent total mastectomy at the breast surgery unit and immediate reconstruction with a prosthesis from March 2017 to September 2018 were enrolled. We retrospectively reviewed the patients' medical records. Patients who underwent bilateral breast reconstruction, those who underwent additional procedures such as contralateral breast manipulations or fat grafting, and those with implants in the prepectoral plane were excluded. We recorded patients' age, body mass index (BMI), the presence of hypertension and diabetes mellitus, smoking history, breast ptosis grade, mastectomy type (nipplesparing mastectomy or total mastectomy), axillary operation status, neoadjuvant treatment, breast specimen weight, and reconstruction type (tissue expander or direct-to-implant). Two general surgeons performed the mastectomies, and the reconstructions were performed by a single plastic surgeon. From March 2017 to January 2018, patients underwent surgery with a monopolar electrosurgical device (group 1) and from February 2018 to September 2018, an ultrasonic dissection device was used (group 2).

\section{Surgical technique}

After total mastectomy was performed by the breast surgeon, immediate breast reconstruction was performed by the plastic surgeon. First, we performed hemostasis in the whole surgical field using an ultrasonic dissection device (Harmonic; Ethicon, Cincinnati, $\mathrm{OH}, \mathrm{USA}$ ). The origin of the pectoralis major muscle was dissected and elevated using the instrument (Fig. 1). After the muscle was adequately elevated, we made a slit incision on a piece of acellular dermal matrix (ADM), which had been immersed in an antibiotic solution, and fixed the $\mathrm{ADM}$ in the new inframammary fold location using Vicryl 3-0 sutures. We then fixed the edges of the pectoralis major muscle with the $\mathrm{ADM}$ to create a pocket where the mammary implant or tissue expander would be inserted. A 400-mL Hemovac was inserted in the margin of the breast pocket. We copiously irrigated the breast pocket with a triple antibiotic solution (cefazolin, gentamycin, and metronidazole). After we applied amorphous betadine on the breast skin, we changed into aseptic gloves and inserted the mammary implant (or tissue expander) into the breast pocket. Then, we closed the pocket by suturing the $\mathrm{ADM}$ and the pectoralis major muscle. After subcutaneous suturing, subdermal suturing was performed using the buried technique, and skin closure was performed using 5-0 nylon sutures. A mild compressive dressing was applied, and the operation was completed. Similar surgical techniques were used in the monopolar electrosurgical device group and the ultrasonic scalpel group; only the dissection device was different between the groups. 


\section{Fig. 1. Device and intraoperative image of its use}

\begin{abstract}
(A) Harmonic Focus, ultrasonic shears, curved tip (Ethicon EndoSurgery, Inc., Cincinnati, OH, USA). $(B, C)$ During the ultrasonic dissection to elevate the pectoralis major muscle, there was no muscle contraction, and hemostasis was straightforward. Furthermore, there was less thermal damage to the nearby tissue.
\end{abstract}

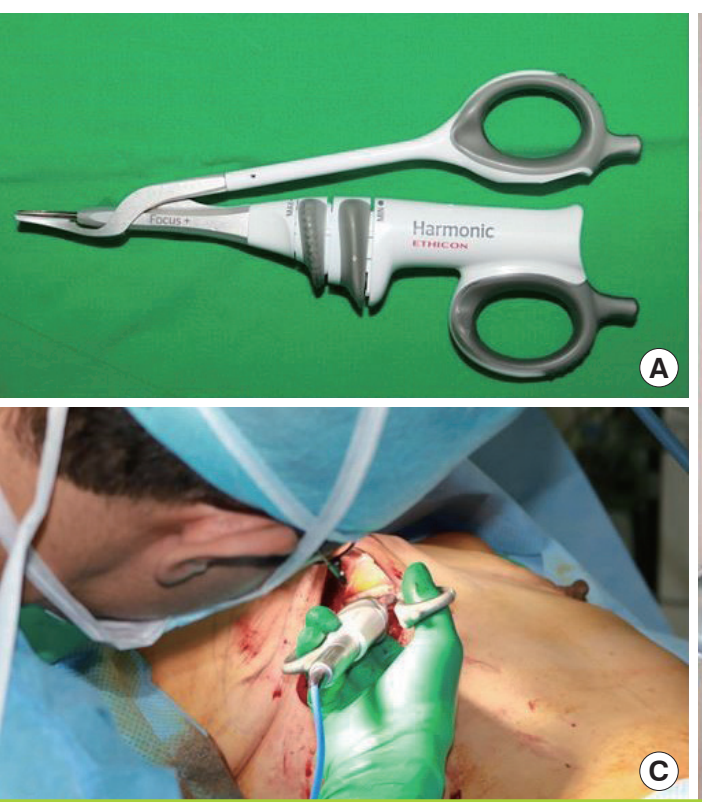

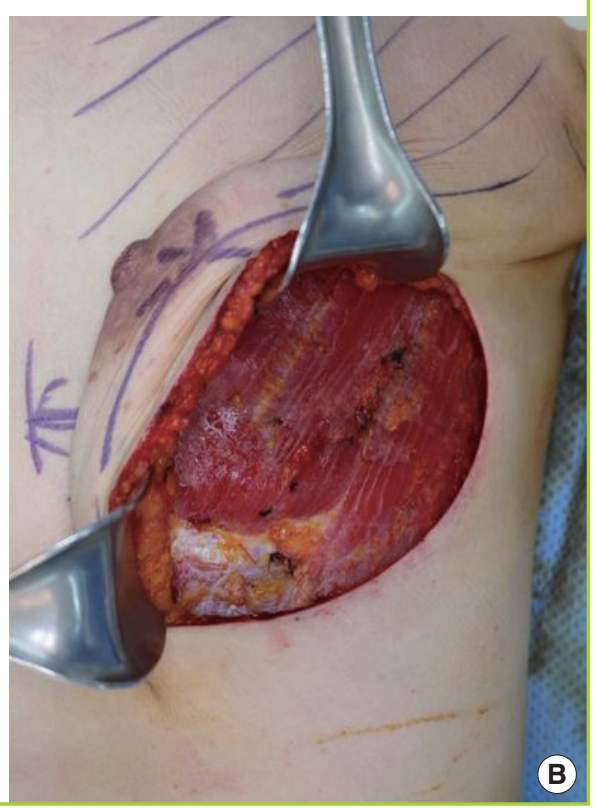

\section{Outcome variables}

The outcome variables were duration of surgery (minutes), drain volume $(\mathrm{mL})$, duration of drainage (days) and postoperative complications (e.g., seroma, hematoma, flap necrosis, or surgical site infection). Seroma was defined as the presence of a fluid collection within 30 days of surgery beneath the skin flaps after drain removal, in sufficient quantity to cause the patient discomfort. Surgical site infections were assessed using the Centers for Disease Control and Prevention criteria, according to which an SSI is defined as an infection of the skin and subcutaneous tissue that occurs within 30 days postoperatively and satisfies at least one of the following criteria: (1) purulent drainage from the superficial incision; (2) organisms (other than Staphylococcus epidermidis) isolated from an aseptically obtained culture of fluid or tissue from the superficial incision; (3) at least one of the given signs or symptoms of infection (i.e., pain or tenderness; localized swelling; redness; heat; superficial incision deliberately opened by surgeon and was culture-positive or not cultured [a culture-negative finding does not meet this criterion]); and (4) diagnosis of a superficial incisional surgical site infection by the surgeon or attending physician. Hematoma was defined as the need to perform aspiration or surgical evacuation due to the collection of blood under the flap. Flap necrosis was defined as the need to perform surgical revision due to a flap wound that occurred after surgery.

\section{Statistical analysis}

Statistical analysis was conducted using SPSS version 23.0 (IBM Corp., Armonk, NY, USA). Baseline demographic characteris-

\begin{tabular}{|c|c|c|c|}
\hline Variable & Group 1 & Group 2 & P-value \\
\hline Age (yr) & $47.6 \pm 7.0$ & $45.1 \pm 6.8$ & 0.052 \\
\hline BMI $\left(\mathrm{kg} / \mathrm{m}^{2}\right)$ & $23.3 \pm 2.8$ & $22.4 \pm 2.8$ & 0.089 \\
\hline \multicolumn{4}{|l|}{ Comorbidity } \\
\hline DM & $4(6.3)$ & $1(1.7)$ & 0.368 \\
\hline HTN & $1(1.6)$ & $1(1.7)$ & $>0.999$ \\
\hline Smoking & $1(1.6)$ & $3(5.2)$ & 0.345 \\
\hline Ptosis grade & & & 0.401 \\
\hline 0 & $26(40.6)$ & 32 (55.2) & \\
\hline 1 & 24 (37.5) & 17 (29.3) & \\
\hline 2 & $9(14.1)$ & 7 (12.1) & \\
\hline 3 & $5(7.8)$ & $2(3.4)$ & \\
\hline
\end{tabular}

Values are presented as mean \pm SD or number (\%).

Group 1, monopolar electrosurgical device group; group 2, ultrasonic dissection device group; BMI, body mass index; DM, diabetes mellitus; HTN, hypertension.

tics (Table 1), treatment information (Table 2), and outcomes (Tables 3, 4) were compared using the Student t-test, chi-square test, and Fisher exact test according to the intervention device. Univariate and multivariate analyses were performed using logistic regression to analyze the effects of the variables in each group. The multivariate analysis was performed using variables identified as affecting seroma development in the univariate analysis (intervention device, age, weight of specimen, BMI, and reconstruction type).

\section{RESULTS}

The monopolar electrosurgical device group (group 1) comprised 58 breasts, while the ultrasonic dissection device group 


\section{Table 2. Treatment information}

\begin{tabular}{|lccc|}
\hline Variable & Group 1 & Group 2 & P-value \\
\hline Type of mastectomy & & & 0.379 \\
Nipple-sparing mastectomy & $27(42.2)$ & $30(51.7)$ & \\
Skin-sparing mastectomy & $14(21.9)$ & $14(24.1)$ & \\
Total mastectomy & $23(35.9)$ & $14(24.1)$ & \\
Axillary operation & & & 0.679 \\
SLNB & $49(76.6)$ & $42(72.4)$ & \\
ALND & $15(23.6)$ & $16(27.6)$ & \\
Neoadjuvant chemotherapy & $17(26.6)$ & $12(20.7)$ & 0.525 \\
Specimen weight (g) & $460.9 \pm 203.9$ & $356.3 \pm 185.9$ & 0.004 \\
General surgeon & & & 0.425 \\
A & $42(65.6)$ & $34(58.6)$ & \\
B & $22(34.4)$ & $24(41.4)$ & \\
Reconstruction type & & & 0.229 \\
Tissue expander insertion & $40(62.5)$ & $30(51.7)$ & \\
Direct-to-implant & $24(37.5)$ & $28(48.3)$ & \\
\hline Values are presented as number (\%) or mean \pm SD. & & \\
Group 1, monopolar electrosurgical device group; group 2, ultrasonic dissection \\
device group; SLNB, sentinel lymph node biopsy; ALND, axillary lymph node \\
dissection.
\end{tabular}

\section{Table 3. Outcomes of surgery}

\begin{tabular}{|lccc|}
\hline Variable & Group 1 & Group 2 & P-value \\
\hline Duration of surgery (min) & $102.7 \pm 24.4$ & $98.2 \pm 31.7$ & 0.439 \\
Drain volume (mL) & $1,677.7 \pm 639.0$ & $1,762.7 \pm 532.1$ & 0.484 \\
Duration of drainage (day) & $18.9 \pm 4.5$ & $17.8 \pm 3.3$ & 0.205 \\
\hline $\begin{array}{l}\text { Values are presented as mean } \pm \text { SD. } \\
\text { Group 1, monopolar electrosurgical device group; group 2, ultrasonic dissection } \\
\text { device group. }\end{array}$
\end{tabular}

(group 2) comprised 64 breasts. In a comparison of the baseline demographic characteristics of the two groups, we found that the patients in group 2 were older (group 1 vs. group 2: $47.6 \pm$ 7.0 years vs. $45.1 \pm 6.8$ years, $P=0.052)$, and that the average BMI was lower in group 1 (group 1 vs. group 2: $23.3 \pm 2.8 \mathrm{~kg} / \mathrm{m}^{2}$ vs. $22.4 \pm 2.8 \mathrm{~kg} / \mathrm{m}^{2}, \mathrm{P}=0.089$ ). However, neither age nor BMI was statistically significant. Hypertension, diabetes mellitus, smoking, and ptosis grade were also similar between the groups (Table 1 ). In group 2 , the specimen weight was significantly greater (group 1 vs. group 2: $460.9 \pm 203.9$ g vs. $356.3 \pm 185.9$ g, $\mathrm{P}=0.004$ ) We surveyed the types of mastectomy, axillary operation status, chemotherapy, and reconstruction type, and these variables were comparable (Table 2). Furthermore, the duration of surgery, drain volume, and duration of drainage were similar in both groups (Table 3 ).

With respect to surgical outcomes, the seroma development rate was relatively high in group 1 (11 [17.2\%] vs. 18 [31.0\%], $\mathrm{P}=0.090)$. Hematoma did not develop in any of the patients in group 2, but five cases of hematoma development were noted in group $1(5[8.6 \%]$ vs. $0[0.0 \%], \mathrm{P}=0.022)$. The duration of sur-
Table 4. Complications of surgery

\begin{tabular}{|lccc|}
\hline Variable & Group 1 & Group 2 & P-value \\
\hline Seroma & $11(17.2)$ & $18(31.0)$ & 0.090 \\
Hematoma & 0 & $5(8.6)$ & 0.022 \\
Surgical site infection & $2(3.1)$ & $3(5.2)$ & 0.668 \\
Flap necrosis & $5(7.8)$ & $4(6.9)$ & 1.000 \\
Overall complication rate & $14(26.5)$ & $16(39.1)$ & 0.274 \\
\hline $\begin{array}{llll}\text { Values are presented as number (\%). } \\
\text { Group 1, monopolar electrosurgical device group; group 2, ultrasonic dissection } \\
\text { device group. }\end{array}$ \\
\hline
\end{tabular}

Table 5. Multivariate analysis of variables affecting the likelihood of seroma development

\begin{tabular}{|lcc|}
\hline & Adjusted OR (95\% Cl) & P-value \\
\hline Intervention & 0.016 \\
Ultrasonic dissection & 1 (reference) & \\
Electrocautery & $3.252(1.242-8.516)$ & \\
Age & $1.053(0.986-1.125)$ & 0.123 \\
Weight of specimen & $1.002(0.999-1.005)$ & 0.198 \\
BMl & $1.025(0.831-1.265)$ & 0.816 \\
General surgeon & 1 (reference) & 0.174 \\
A & $0.497(0.181-1.363)$ & \\
B & 1 (reference) & 0.143 \\
Reconstruction type & 2.033 (0.787-5.252) \\
Direct-to-implant & \\
Tissue expander & \\
\hline OR, odds ratio; Cl, confidence interval; BMl, body mass index. & \\
\hline
\end{tabular}

gery, total drainage volume, duration of drainage, overall complication rate, surgical site infection rate, and flap necrosis rate were comparable (Table 4).

Univariate analysis was performed to identify the factors affecting the seroma development rate, and we found that the risk of seroma development was higher in group 1 than in group 2 (odds ratio [OR] for electrocautery, 2.168; 95\% CI, $0.922-$ 5.099; $\mathrm{P}=0.076$ ). Multivariate analysis was performed using variables that could clinically affect the occurrence of seroma. Age, BMI, specimen weight, reconstruction type, and intervention type were included in the multivariable analysis; a significantly lower risk of seroma development was observed in the ultrasonic dissection device group (OR for electrocautery, 3.252; 95\% CI, 1.242-8.516; $\mathrm{P}=0.016$ ) (Table 5).

\section{DISCUSSION}

We aimed to compare the effects of using an ultrasonic dissection device and a monopolar electrosurgical device on seroma formation and short-term outcomes in patients undergoing prosthetic breast reconstruction after mastectomy, and found that that the rate of seroma development was lower in the ultra- 
sonic dissection device group (group 2). The incidence of short-term complications was lower in group 2, but this tendency was not statistically significant. There was no statistically significant difference in the operative time between the two groups. The total drainage volume, duration of drainage, surgical site infection rate, and flap necrosis rate were also comparable between the two groups.

Ultrasonic dissection devices have been reported to be superior to monopolar electrosurgical devices in many studies. They provide the advantages of better hemostasis with minimal thermal damage, a reduced risk of nerve damage, the use of fewer instruments due to their combined vessel-sealing, tissue-cutting, and tissue-dissecting functionality, and less visual obstruction from smoke [13-16]. Using the principle of breaking hydrogen bonds due to the generation of ultrasonic energy, these devices dissect the tissue and seal the vessels and lymphatics. This method was first used in minimally invasive surgery, including laparoscopic surgery; since then, it has been gradually proven to be superior to traditional dissection techniques, as shown by a decreased incidence of postoperative complications, including a lower rate of seroma development. The superiority of ultrasonic dissection in breast surgery remains controversial, but a report has described good outcomes for a recently developed modified radical mastectomy technique using an ultrasonic dissection device compared to those reported for mastectomy performed using a monopolar electrosurgical device [10].

Despite being a retrospective study, this study was the first to compare the short-term complications of surgery using a monopolar electrocautery device and an ultrasonic dissection device in immediate implant-based breast reconstruction. Cases in which factors other than the tools used in the intervention could have affected surgical outcomes were excluded from this study. In addition, recommendations of ways to reduce the complication rate in prosthetic breast reconstruction, such as using $\mathrm{ADM}$, irrigation of the breast pocket with antibiotic solution, and the surgeon wearing sterile gloves before insertion of the implant, were applied in the surgical procedures presented herein.

Seroma formation can be caused by many factors. Currently, the leading causes of seroma formation are thought to be persistent inflammation after mastectomy, thermal damage to tissue, and lymphatic disruption. In this study, the use of an ultrasonic dissection device decreased the seroma formation rate. It seems that when elevating the pectoralis major muscle, there was less thermal damage of the anterior chest wall and muscle, which reduced the formation of seroma due to inflammation. In the hemostasis process, the ultrasonic scalpel is applied only to selective areas, unlike when a monopolar electrosurgical device is used; therefore, tissue damage is markedly reduced in the overall area of the surgical field.

In addition to a reduced incidence of seroma development, ultrasonic dissection devices have many other advantages. Charring, which occurs when using a monopolar electrosurgical device for muscle elevation, is not produced with this device. Charring can be a risk factor for prosthesis-related infection because the charred substance is not entirely removed during the irrigation performed before inserting an implant. Therefore, direct contact between the charred substance and the prosthesis is eliminated, thereby reducing the likelihood of infection. The need to administer a muscle relaxant is eliminated because ultrasonic dissection devices do not cause muscle contractions. They also reduce the possibility of anesthesia-related complications, such as anaphylaxis, and prevent an unnecessary prolongation of anesthesia time.

There are several limitations of this study. First, the sample size of the two groups was not sufficiently large, which may explain the presence of between-group differences in specimen weight and BMI. However, a sufficiently large sample size would be expected to eliminate such differences between the two groups. Of particular note, a statistically significant difference was noted for specimen weight (group 1 vs. group 2: $460.9 \pm 203.9 \mathrm{~g}$ vs. $356.3 \pm 185.9 \mathrm{~g}, \mathrm{P}=0.004)$. However, higher-weight specimens are generally associated with increased seroma incidence and drainage volume. Therefore, the multivariate analysis adjusted for the possible effects of differences in specimen weight on the incidence of seroma in the monopolar electrosurgical group. Second, the general surgeons did not use the ultrasonic dissection device in a way consistent with how the plastic surgeon did so. The surgical technique used in mastectomy is expected to have a significant effect on seroma formation after reconstruction.

In conclusion, the rate of seroma formation can be slightly reduced by using an ultrasonic dissection device for immediate implant-based breast reconstruction. However, further randomized controlled studies are required to verify our results and to assess the cost-effectiveness of this technique.

\section{NOTES}

\section{Conflict of interest}

No potential conflict of interest relevant to this article was reported.

\section{Ethical approval}

The study was approved by the Institutional Review Board of Gangnam Severance Hospital (IRB No. 3-2019-0266) and performed in accordance with the principles of the Declaration of Helsinki. 


\section{Patient consent}

The patient provided written informed consent for the publication and the use of her images.

\section{Author contribution}

Conceptualization: Kim YS. Data curation: Kim YS, Roh TS. Formal analysis: Lee DE, Jung BK. Funding acquisition: Kim YS. Methodology: Kim YS. Project administration: Kim YS. Visualization: Lee DE. Writing - original draft: Lee DE. Writing review \& editing: Kim YS. Approval of final manuscript: all authors.

\section{ORCID}

Dongeun Lee https://orcid.org/0000-0001-5180-0495

Bok Ki Jung https://orcid.org/0000-0002-4347-560X

Tai Suk Roh https://orcid.org/0000-0001-8681-159X

Young Seok Kim https://orcid.org/0000-0002-0981-2107

\section{REFERENCES}

1. Woodworth PA, McBoyle MF, Helmer SD, et al. Seroma formation after breast cancer surgery: incidence and predicting factors. Am Surg 2000;66:444-50.

2. Di Monta G, Caraco C, Crispo A, et al. Collagen sealant patch to reduce lymphatic drainage after lymph node dissection. World J Surg Oncol 2012;10:275.

3. McCaul JA, Aslaam A, Spooner RJ, et al. Aetiology of seroma formation in patients undergoing surgery for breast cancer. Breast 2000;9:144-8.

4. Srivastava V, Basu S, Shukla VK. Seroma formation after breast cancer surgery: what we have learned in the last two decades. J Breast Cancer 2012;15:373-80.

5. Yilmaz KB, Dogan L, Nalbant H, et al. Comparing scalpel, electrocautery and ultrasonic dissector effects: the impact on wound complications and pro-inflammatory cytokine levels in wound fluid from mastectomy patients. J Breast
Cancer 2011;14:58-63.

6. Kuroi K, Shimozuma K, Taguchi T, et al. Pathophysiology of seroma in breast cancer. Breast Cancer 2005;12:288-93.

7. Odell RC. Electrosurgery: principles and safety issues. Clin Obstet Gynecol 1995;38:610-21.

8. Porter KA, O'Connor S, Rimm E, et al. Electrocautery as a factor in seroma formation following mastectomy. Am J Surg 1998;176:8-11.

9. Faisal M, Fathy H, Shaban H, et al. A novel technique of harmonic tissue dissection reduces seroma formation after modified radical mastectomy compared to conventional electrocautery: a single-blind randomized controlled trial. Patient Saf Surg 2018;12:8.

10. Huang J, Yu Y, Wei C, et al. Harmonic scalpel versus electrocautery dissection in modified radical mastectomy for breast cancer: a meta-analysis. PLoS One 2015;10:e0142271.

11. He Q, Zhuang D, Zheng L, et al. Harmonic focus versus electrocautery in axillary lymph node dissection for breast cancer: a randomized clinical study. Clin Breast Cancer 2012;12: 454-8.

12. Khan S, Khan S, Chawla T, et al. Harmonic scalpel versus electrocautery dissection in modified radical mastectomy: a randomized controlled trial. Ann Surg Oncol 2014;21:80814.

13. Devassy R, Gopalakrishnan S, De Wilde RL. Surgical efficacy among laparoscopic ultrasonic dissectors: are we advancing safely? A review of literature. J Obstet Gynaecol India 2015;65:293-300.

14. Law KS, Abbott JA, Lyons SD. Energy sources for gynecologic laparoscopic surgery: a review of the literature. Obstet Gynecol Surv 2014;69:763-76.

15. Wang K, Advincula AP. "Current thoughts" in electrosurgery. Int J Gynaecol Obstet 2007;97:245-50.

16. Lyons SD, Law KS. Laparoscopic vessel sealing technologies. J Minim Invasive Gynecol 2013;20:301-7. 